\title{
Connection between primary Fusarium inoculum on gramineous weeds, crop residues and soil samples and the final population on wheat ears in Flanders
}

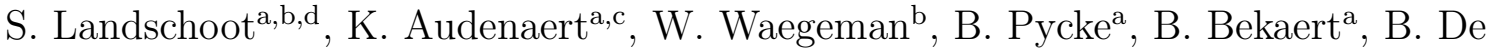 \\ Baets $^{\mathrm{b}}$, G. Haesaert ${ }^{\mathrm{a}, \mathrm{c}}$ \\ ${ }^{a}$ Department of Biological Sciences and Landscape Architecture, University College Ghent, \\ Schoonmeersstraat 52, BE-9000 Ghent, Belgium, Fax: 0032 92424293 \\ ${ }^{b}$ KERMIT, Department of Applied Mathematics, Biometrics and Process Control, Ghent University, \\ Coupure links 653, BE-9000 Ghent, Belgium \\ ${ }^{c}$ Department of Crop Protection, Laboratory Phytopathology, Ghent University, Coupure links 653, \\ BE-9000 Ghent, Belgium \\ ${ }^{d}$ corresponding author: Sofie Landschoot, E-mail: sofie.landschoot@hogent.be, Tel: +32 (0)9248 88 60, \\ Fax: +32 (0)9 242 42 79
}

\begin{abstract}
Fusarium head blight (FHB) is a devastating disease of wheat, which is influenced by weather conditions and agronomic factors. Since FHB is a mostly monocyclic disease, the quantity of primary inoculum is a key factor influencing the FHB incidence. To investigate the connection between the primary Fusarium inoculum and the final population on wheat ears, naturally occurring populations of Fusarium avenaceum, Fusarium culmorum, Fusarium graminearum, Fusarium poae and Microdochium nivale were studied at eight locations in Flanders, Belgium. To determine the composition of the primary inoculum in November 2008, gramineous weeds, wheat residues, maize residues and soil samples were examined. In July 2009, wheat ears were examined to gain insight into the composition of the population at the end of the growing season. Comparing both populations can give an indication to what extent the primary inoculum in November 2008 contributes to the final FHB population in July 2009. Fusarium species residing saprophytically on gramineous weeds and crop residues or species persisting in soil as chlamydospores certainly contribute to the primary inoculum that will lead to infection of cereal crops grown on that field. Knowledge of the correlation between the primary inoculum and the composition of the FHB population at the end of the growing season can be important to predict the occurrence of FHB contamination and to implement control strategies for Fusarium Head Blight.
\end{abstract}

Keywords:

Fusarium Head blight, primary inoculum, winter wheat 


\section{Introduction}

Fusarium Head Blight (FHB) is one of the most important diseases in small grain cereals, caused by a complex of Fusarium species. Although FHB may cause grain yield losses, the interest in FHB is primarily fueled by the ability of the majority of the Fusarium species to produce mycotoxins. These secondary fungal metabolites can accumulate to significant doses and as such cause a serious impediment for human and animal health. Moreover, European legislation for several Fusarium mycotoxins has been concretized in regulations for maximum levels for human and animal consumption. These regulations provide an extra economic motive for farmers to prevent FHB infection and mycotoxin accumulation in small grain cereals such as wheat. Until now, 17 species of Fusarium have been described to be potentially associated with FHB symptoms (Leonard and Bushnell, 2003). The main causal agents of FHB in Europe are Fusarium graminearum, Fusarium culmorum, Fusarium avenaceum, Fusarium poae and Microdochium nivale. F. graminearum, F. culmorum, $F$. avenaceum and $F$. poae can produce a plethora of mycotoxins, whereas apparently M. nivale does not produce mycotoxins (Xu et al., 2005).

Recently, a fast growing number of reports are describing the increasing importance of other species such as F. langsethiae, which was previously not associated with FHB in Europe (Lukanowski et al., 2008; Torp and Nirenberg, 2004). Many laboratories have devoted considerable effort to unequivocally delineate the Fusarium population, but the dynamics within the population seems inexhaustible. Results obtained by Waalwijk et al. (2003) in The Netherlands illustrate the highly dynamic nature of the FHB population with shifts from F. culmorum in the early 1990s to F. graminearum in 2000 and 2001. For Flanders a similar phenomenon was described by Isebaert et al. (2009) and Audenaert et al. (2009), illustrating a shift from a population dominated by F. graminearum and $F$. culmorum to a population merely consisting of $F$. poae. Analogous results were obtained in Fusarium surveys throughout Europe (Kosiak et al., 2003; Köhl et al., 2007). Besides these interseasonal variations, $\mathrm{Xu}$ et al. (2005) demonstrated the increasing complexity of the population during one growing season from anthesis to harvest by the increasing number of FHB pathogens and the increase of interactions between species. The dynamic nature of the population and the various factors that contribute to this complexity are a serious hindrance to controlling and forecasting FHB during the growing season.

The distribution and predominance of FHB pathogens is to a large extent determined by climatic factors, in particular temperature and moisture. However, under the European climate, these are not the sole variables determining the population's composition. Crop husbandry practices also have an important influence on the predominance of FHB pathogens. Fungicide treatment, crop rotation system, weed management, host resistance and soil tillage are probably the most important agricultural factors governing the out- 
come and structure of the FHB population (Schaafsma and Hooker, 2007; Koch et al. 2006). During previous years major changes could be observed in these crop husbandry practices. Soil tillage systems shifted to minimum or zero tillage systems to sustain good soil structure and weed management was redirected to increase ecological diversity by a modified mowing strategy of the surrounding verges. The impact of these measures on the occurrence of FHB remains to be determined and a structured view on the impact of reduced tillage measures and the abundance of gramineous weeds on the composition of primary inoculum are beyond the frontiers of current knowledge. However, reduced tillage measures combined with crop residues overwintering on the field certainly contribute to the primary inoculum in the following cereal crop in spring, since head blight epidemics are mostly monocyclic. The primary reservoir of inoculum is debris from the previous crop. All species of Fusarium and Microdochium can survive as saprophytes. Regarding gramineous weeds it is known that most Fusarium species have a broad spectrum of hosts among gramineous weeds. Fusarium species residing saprophytically on gramineous weeds could therefore contribute to the primary inoculum. Fusarium spp. and M. nivale infect wheat ears primarily during anthesis. Chances for infection depend on many factors, including the amount of spores produced in the crop residues and transported to the wheat ears, weather conditions, and susceptibility of the cultivar. The main sources of inoculum consist of splashdispersed conidia that originate from infected residues of previous crops still present in the field. A study by Pereyra and Dill-Macky (2008) showed that F. graminearum isolates surviving during winter on weeds such as Lolium, Digitaria and Setaria were also virulent on wheat and barley, which illustrates the potential of these weed residing populations in the FHB disease development. This study also illustrated discrepancies in survival capacities between several species such as $F$. graminearum and $F$. poae. F. graminearum, M. nivale and F. avenaceum are also able to produce ascospores, which contribute to the local inoculum but also may travel longer distances, so that airborne inoculum produced outside the field may also initiate disease (Köhl et al., 2007). Finally, some Fusarium species can also persist in soil as saphrophytic mycelium or as thick-walled resting spores (chlamydospores). Soil-borne infections take hold less rapidly than other infections and resulting attacks affect essentially the collar and the upper parts of the roots (Champeil et al. 2004).

In this particular study the connection between the primary Fusarium inoculum on weeds, crop residues and soil samples and the final FHB population on wheat ears was investigated in Flanders during the growing season 2008-2009. Under low infection pressure, a direct relationship between the diversity of inoculum and the disease incidence can be expected because head blight epidemics are mostly monocyclic: the pathogen has only one infection cycle per season (Köhl et al., 2007). Knowledge of the correlation between the primary Fusarium inoculum and the FHB population can be important to develop a prediction 
model for FHB, which is the overall objective of our study.

\section{Materials and methods}

\subsection{Sampling during the growing season}

In order to gain insight into the Fusarium population during the growing season 20082009, samples were taken at locations Bottelare, Koksijde, Linter, Poperinge, Tongeren, Verrebroek, Zwalm and Zwevegem. These locations are distributed throughout the most important wheat regions in Flanders, and are part of the LCG trial network (Landbouw Centrum Granen (Agricultural Center Cereals), Roeselare-Beitem, Belgium). At each location, 12 commercial winter wheat cultivars were laid out as completely randomized block design with $15 \mathrm{~m}^{2}$ plots and four replications. At all locations wheat was produced under normal crop husbandry conditions for Flanders, i.e. ploughing, 350 kernels $/ \mathrm{m}^{2}$ as sowing density, three split $\mathrm{N}$-fertilization and one or two fungicide treatments applied at growth stage (GS) 39 and 59, respectively. To study the composition of the primary inoculum, at each location soil samples, crop residues and weed samples from the field and its surrounding verges were taken at the time of sowing winter wheat, in November and December 2008, depending on the sowing date.

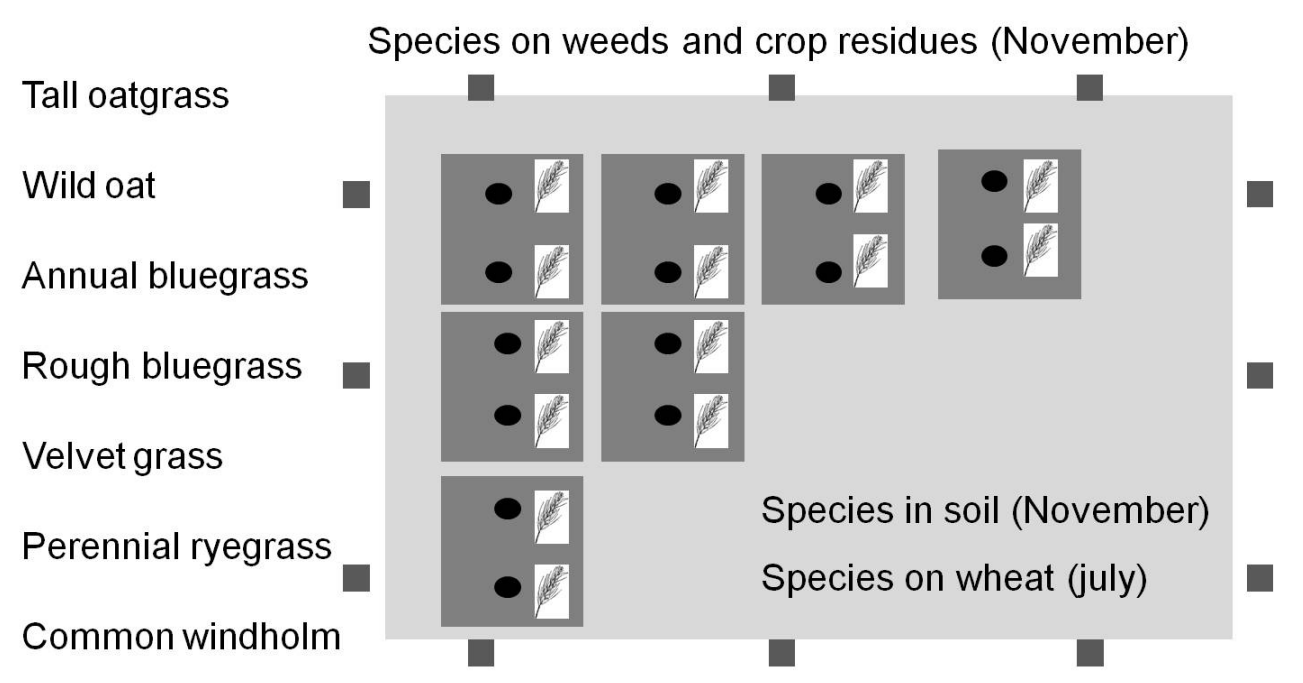

Figure 1: Sample scheme of soil, weeds, crop residues and wheat ears. The large gray rectangle shows the field trial, the three rows of dark gray rectangles are the $15 \mathrm{~m}^{2}$ plots of the wheat cultivars and the two black circles in each of these rectangles show the soil samples per plot. Weeds and crop residues were sampled along the sides of the field trial, the positions are indicated with dark gray squares. In July two wheat ears from each plot were harvested.

Figure 1 shows the sample scheme of soil, gramineous weeds, crop residues and wheat ears. The circles show the two soil samples per plot. Weeds and crop residues were sampled 
along the sides of the field; the positions are indicated with squares. A range of gramineous weeds, which act as alternative hosts for Fusarium species, were determined using Weeda et al. (1983). These are Italian ryegrass (Lolium mulitflorum), perennial ryegrass (Lolium perenne), wild oat (Avena fatua), annual bluegrass (Poa annua), rough meadow grass (Poa trivialis), velvet grass (Holcus lanatus), cocksfoot (Dactylis glomerata), loose silky-bent (Apera spica venti), quack grass (Elytrigia repens) and common bent (Agrostis cappilaris). The crop residues include maize and wheat residues, since these are host plants on which Fusarium species can survive saprophytically.

To determine the composition of the Fusarium population during the growing season, samples were taken between at the beginning of July at GS 71 (watery ripe) or GS 75 (milky ripe). At each location, two symptomatic ears from each variety and each parallel were harvested. From each ear, two symptomatic kernels were isolated for further identification.

\subsection{Plating experiments}

The soil samples were air-dried and stored at $4{ }^{\circ} \mathrm{C}$ until processing. Subsamples of the soil $(10 \mathrm{~g}$ each) were added to $100 \mathrm{ml}$ water and mixed thoroughly. One hundred $\mu \mathrm{l}$ of the final dilution was transferred to petri dishes containing dichloran-diglycerol (DG18) agar (Oxoid, Belgium) with 2.5mg/l Malachite Green Agar (MGA 2.5) and $300 \mathrm{mg} / \mathrm{l}$ chloramphenicol. MGA combined with antibacterial agents is a selective medium for isolation of Fusarium species (Castellá et al., 1997). The petri dishes were incubated at $20^{\circ} \mathrm{C}$, since at this temperature F. avenaceum, F. culmorum, F. graminearum, F. poae and M. nivale are able to grow in vitro (Hudec and Muchová, 2010, Brennan et al., 2003). All Fusarium colonies were transferred to PDA (potato dextrose agar, Oxoid, Belgium) medium for further identification.

Weeds, crop residues and seeds were surface-sterilized for 1 minute in $1 \% \mathrm{NaOCl}$, washed for 1 minute with $70 \% \mathrm{EtOH}$, washed with distilled sterile water, dried for 5 minutes and subsequently put on PDA plates. After five days of incubation at $20^{\circ} \mathrm{C}$, outgrowing mycelium was transferred to a new PDA plate.

\subsection{Species determination by a species-specific PCR}

For species determination, five mycelium plugs randomly taken from the fully grown PDA plates (section 2.2) were transferred to liquid GPY-broth (10 g glucose, $1 \mathrm{~g}$ yeast extract and $1 \mathrm{~g}$ peptone, Oxoid, Belgium) and incubated for five days at $20^{\circ} \mathrm{C}$. After five days, mycelium was transferred to eppendorf tubes, centrifuged for 10 minutes at $12000 \mathrm{rpm}$ and then freeze-dried for $6 \mathrm{~h}$ at $-10^{\circ} \mathrm{C}$ and $4 \mathrm{~h}$ at $-50^{\circ} \mathrm{C}$ (Christ Alpha 1-2 LD Plus, Osterode, Deutschland). DNA extraction was performed as described by Audenaert et al. (2009) based on the CTAB (hexadecyl trimethyl ammonium bromide) method described by Saghai-Maroof et al. (1984). PCR for single species detection was performed in a $25 \mu \mathrm{l}$ reaction mixture (Demeke et al., 2005). 
DNA amplification was performed in an Applied Biosystems GeneAmp PCR system 97000 PCR. Amplicons were separated on $1.5 \%$ (wt/vol) agarose gels stained with $0.1 \mu$ ethidium bromide. PCR was validated by including reference strains obtained from the MUCL/BCCM collection in each PCR run: Fusarium graminearum MUCL 42841; Fusarium culmorum MUCL 555; Fusarium poae MUCL 6114; Microdochium nivale MUCL 15949; Fusarium avenaceum MUCL 6130 (Audenaert et al., 2009; Isebaert et al., 2009).

\subsection{Statistical analysis}

A chi-squared test was used to compare the incidence of the main Fusarium spp. across locations. Relationships between the species on weeds, residues and wheat were investigated using the Pearson product moment correlation at a significance level of $p=0.05$. All data were analyzed using the software R (version 2.10.1).

\section{Results}

Table 1 shows the overall percentage composition of the primary inoculum on weeds, crop residues and in soil at the time of sowing winter wheat (November 2008) and the FHB population at harvest in July 2009. The dominant species are indicated in bold. The prevalence of Fusarium spp. differed significantly between weed samples, crop residues, soil samples in November 2008 and wheat samples in July 2009. Weed samples were dominated by $M$. nivale, followed by F. graminearum, F. avenaceum, F. culmorum and F. poae, while crop residues were dominated by $F$. culmorum, followed by F. graminearum, $F$. avenaceum, $F$. poae and $M$. nivale. Soil samples were clearly characterized by $F$. culmorum; only a few soil samples contained F. graminearum.

In July 2009, F graminearum was most frequently encountered on wheat samples, followed by $F$. avenaceum, $F$. culmorum and $F$. poae. M. nivale was rarely detected on wheat samples.

Table 1: Overall composition (\%) of primary inoculum on weed samples, crop residues and soil samples in November 2008 and the final Fusarium population on wheat ears in July 2009

\begin{tabular}{lrrrrr}
\hline & F. avenaceum & F. culmorum & F.graminearum & M. nivale & F. poae \\
\hline Gramineous weeds (2008) & 20.93 & 17.48 & 21.686 & $\mathbf{3 5 . 6 6}$ & 4.89 \\
Crop residues (2008) & 11.11 & $\mathbf{3 7 . 7 8}$ & 33.33 & 6.67 & 11.11 \\
Soil (2008) & 0.00 & $\mathbf{9 9 . 1 1}$ & 0.89 & 0.00 & 0.00 \\
Wheat (2009) & 12.50 & 20.83 & $\mathbf{6 1 . 9 8}$ & 0.52 & 4.17 \\
\hline
\end{tabular}




\begin{tabular}{|c|c|c|}
\hline हี & 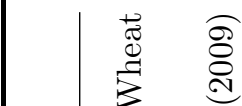 & 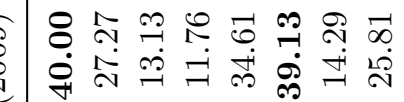 \\
\hline$\overbrace{}^{3}$ & 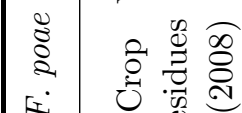 & 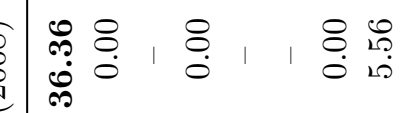 \\
\hline 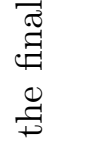 & 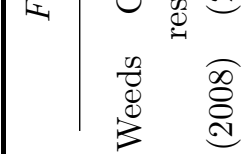 & 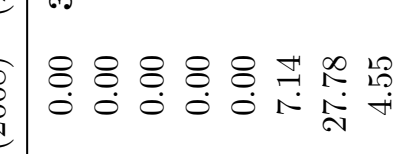 \\
\hline$\infty$ & 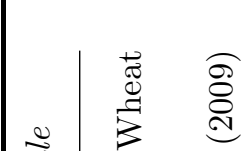 & 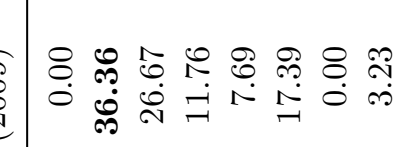 \\
\hline $\begin{array}{l}\stackrel{0}{0} \\
\text { है }\end{array}$ & 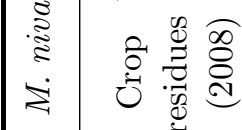 & 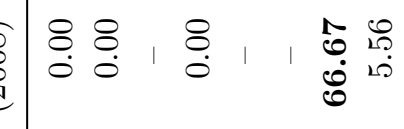 \\
\hline $\begin{array}{l}Z \\
. \nexists\end{array}$ & 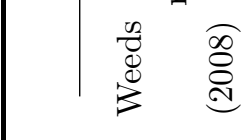 & 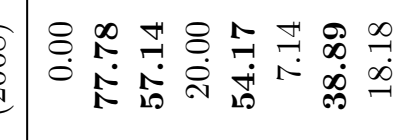 \\
\hline : & 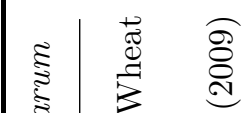 & 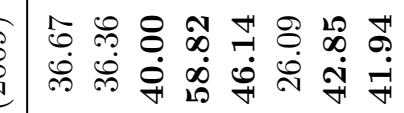 \\
\hline $\overrightarrow{\vec{U}}$ & 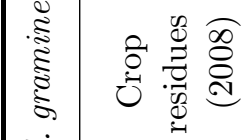 & 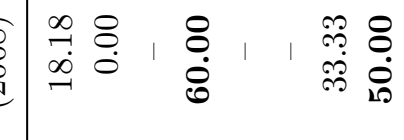 \\
\hline 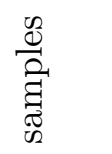 & 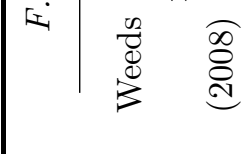 & 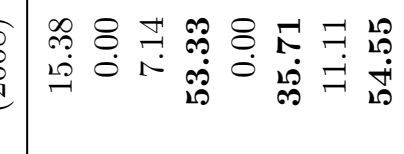 \\
\hline 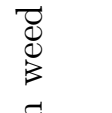 & 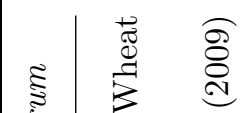 & 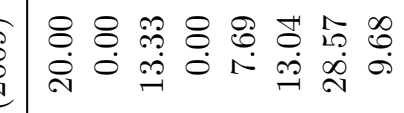 \\
\hline$\stackrel{\circ}{\Xi}$ & 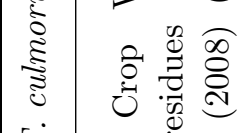 & 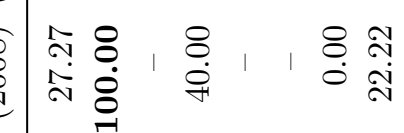 \\
\hline 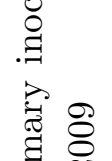 & $\stackrel{\substack{n \\
\mathbb{d}}}{\stackrel{\infty}{0}}$ & 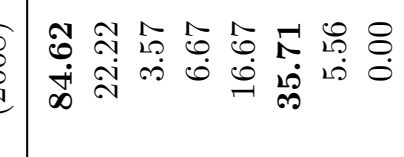 \\
\hline 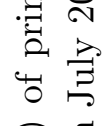 & 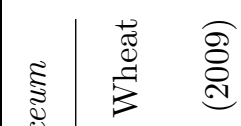 & 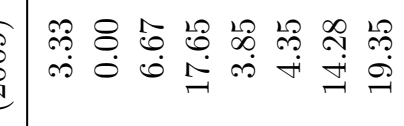 \\
\hline 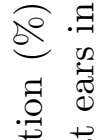 & 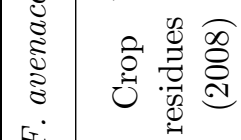 & 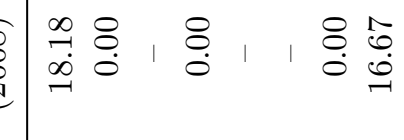 \\
\hline 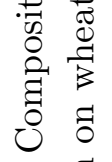 & 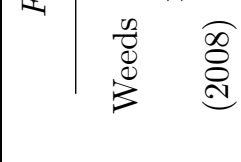 & 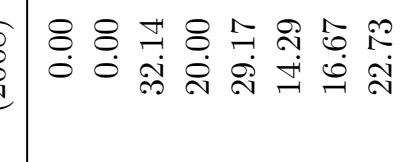 \\
\hline 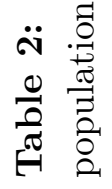 & & 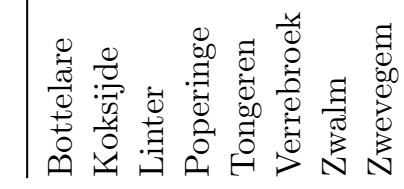 \\
\hline
\end{tabular}




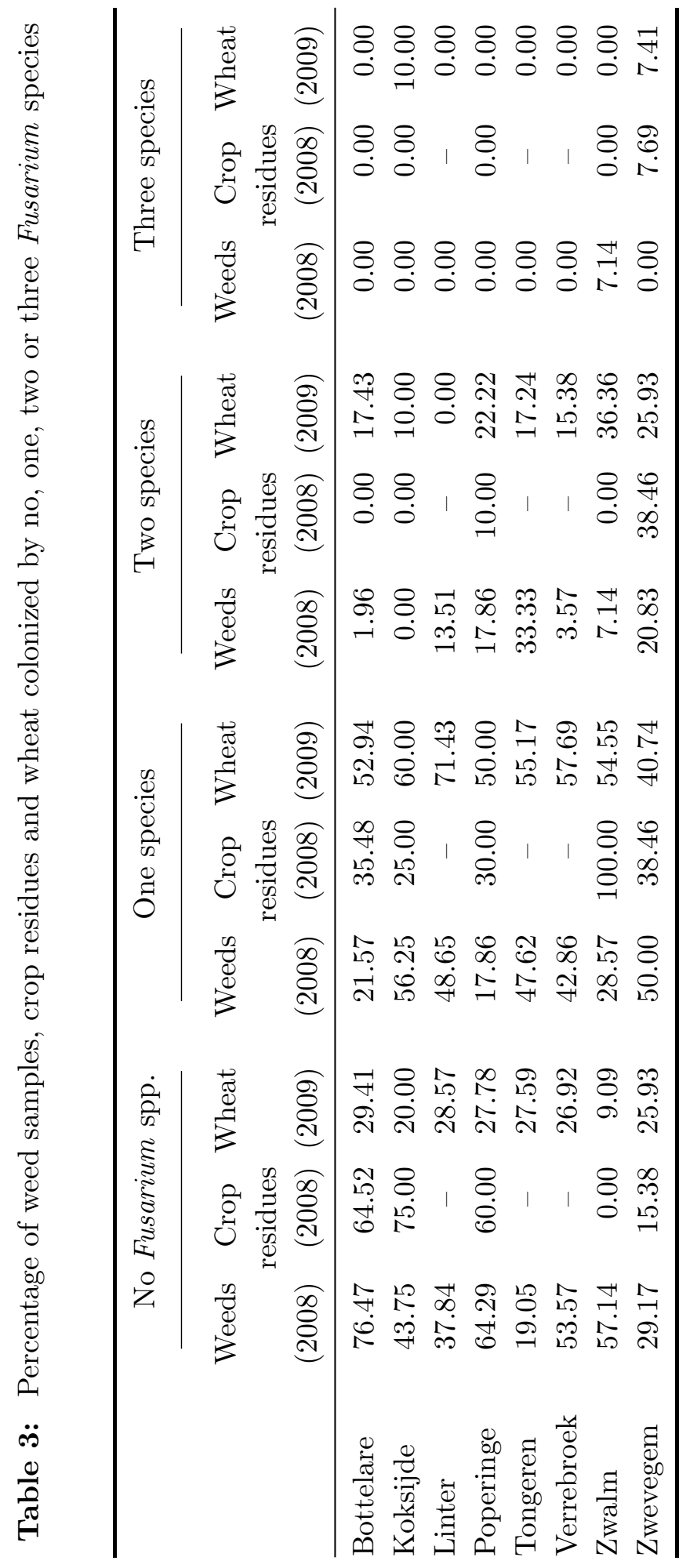


Table 2 shows the composition of primary inoculum on weed samples, crop residues in November 2008 and the population on wheat ears in July 2009 per location. The dominant species at each location is indicated in bold. The missing values for Linter, Tongeren and Verrebroek in Table 2 are due to the fact that at these locations no crop residues were present. The incidence of the five studied Fusarium pathogens on weed samples, crop residues and wheat ears varied considerably between locations (chi-square test: $p<0.05$ ).

In November 2008, in Bottelare and Koksijde only two different species were detected on weed samples, respectively $F$. culmorum-F. graminearum and $F$. culmorum-M. nivale. At other locations three or more different species were found on weeds. F. culmorum was the only species recovered from crop residues in Koksijde. In Poperinge $F$. graminearum and F. culmorum were detected on crop residues and in Zwalm M. nivale and F. graminearum. As in weed samples, crop residues in Zwevegem were colonized by five different species. F. culmorum was the only species recovered from soil samples, except in Poperinge, where also $F$. graminearum was found.

Across Flanders F. graminearum was detected at each location on wheat ears just before harvest, its presence varied between $26.09 \%$ (Verrebroek) and $58.82 \%$ (Poperinge). If the populations in the primary inoculum and the FHB population at the end of the season are compared, then there are some important differences. At first $F$. poae was not detected in the primary inoculum of Koksijde, Linter, Poperinge and Tongeren, but in July each field contained more than $10 \% \mathrm{~F}$. poae. So weed samples and crop residues are probably not the origin of the F. poae inoculum. Furthermore, F. culmorum, omnipresent in soil samples, represents only a small part of the final population. F. avenaceum incidence decreased at all locations. The most striking decrease was in Tongeren, from $29.17 \%$ on weeds to $3.85 \%$ on wheat ears. A similar decrease was found for M. nivale (except in Tongeren); in Zwalm M. nivale was even the main species on crop residues (66.67\%), but on wheat ears this species was completey absent. In Poperinge the population in July 2009 on wheat was similar to the population on weeds in November and in Zwevegem the population on wheat was similar to the population on weeds and on crop residues in November 2008. For the other locations, the population in the primary inoculum differs from the FHB population on wheat ears. 
Bottelare

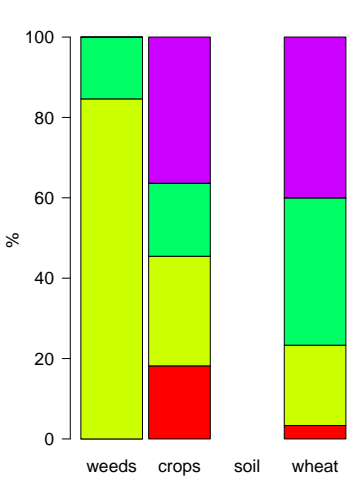

Poperinge

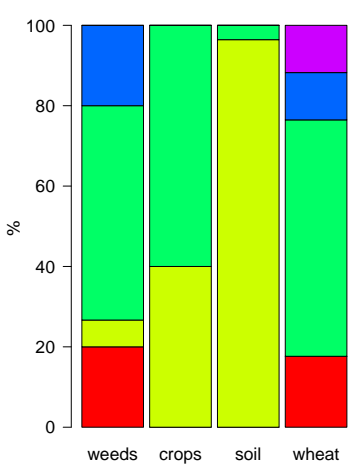

Zwalm

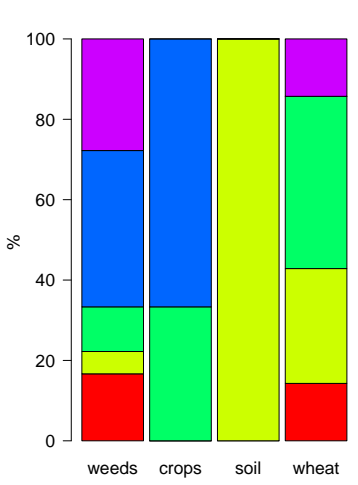

Koksijde

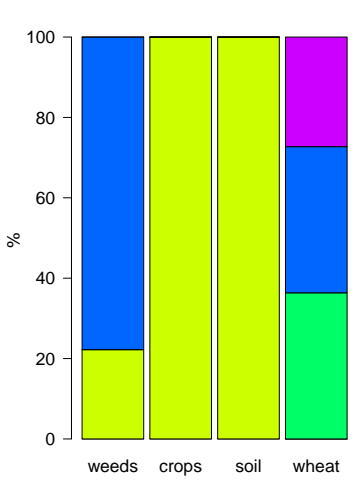

Tongeren

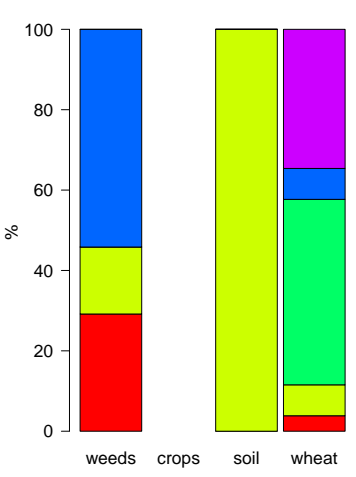

Zwevegem

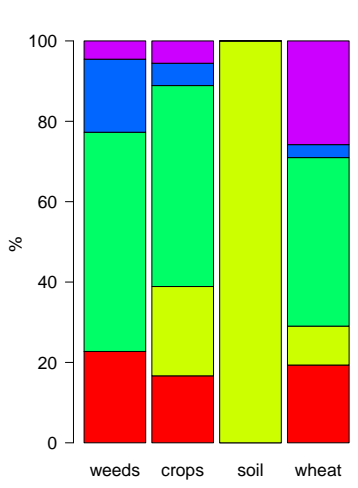

Linter

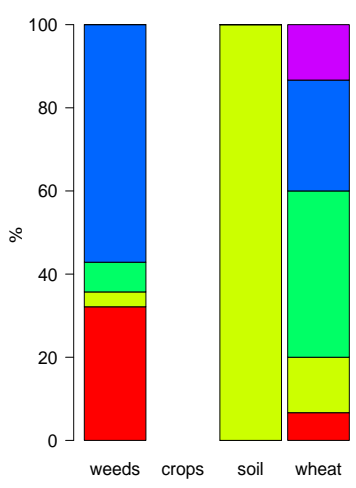

Verrebroek

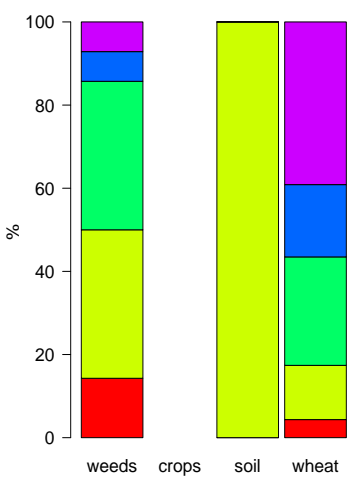

Figure 2: Composition (\%) of primary inoculum on weed samples, crop residues, soil samples at each location in November 2008 and the final Fusarium population in July 2009. 
Table 3 shows the percentage of samples with no, one, two or three different Fusarium species identified. If no species were detected, it does not automatically mean that no Fusarium spp. were present, there may be other species present that were not included in the PCR reaction, e.g. F. langsethiae. However no isolates were observed with typical F. langsethiae morphology.

The majority of the Fusarium spp. appeared as single species. Three species interactions were detected in Zwalm on weed samples (between F. graminearum, M. nivale and F. poae) and in Zwevegem on crop residues (between F. avenaceum, F. culmorum and $F$. graminearum). On wheat ears three species interactions were detected in Zwevegem (between F. avenaceum, F. graminearum and F. poae) and in Koksijde between F. graminearum, M. nivale and F. poae.

Table 4 summarizes the number of samples in which two different species were detected. On weed samples most interactions were detected between $F$. avenaceum and $M$. nivale (eight), whereas on crop residues this interaction was never detected and once on wheat ears. On crop residues the combination F. culmorum-F. graminearum was most frequently observed (four). In wheat ears, the number of samples with F. graminearum and F. poae (16) was clearly higher than the other interactions between two species.

Table 4: Two-species interactions in November 2008

\begin{tabular}{llccc}
\hline \multirow{2}{*}{ Pathogen one } & Pathogen two & \multicolumn{3}{c}{ Number of interactions } \\
\cline { 3 - 5 } & & Weeds & Crop residues & Wheat \\
\hline F. avenaceum & F. culmorum & 0 & 1 & 2 \\
F. avenaceum & F. graminearum & 6 & 2 & 3 \\
F. avenaceum & M. nivale & 8 & 0 & 1 \\
F. avenaceum & F. poae & 2 & 0 & 4 \\
F. culmorum & F. graminearum & 2 & 4 & 5 \\
F. culmorum & M. nivale & 4 & 0 & 0 \\
F. culmorum & F. poae & 0 & 0 & 3 \\
F. graminearum & F. nivale & 5 & 1 & 6 \\
F. graminearum & F. poae & 2 & 1 & 4 \\
M. nivale & F. poae & 3 & 0 & 4 \\
\hline
\end{tabular}




\section{Discussion}

This field survey in Flanders revealed that the Fusarium population varied strongly from location to location, both at the beginning of the season on weeds, crop residues and in soil samples and the end of the season on wheat ears. Regional differences in species profile may exist due to different crop rotation systems and climatic conditions (especially temperature and precipitation) (Waalwijk et al., 2003; Xu et al., 2005).

The dominance of F. culmorum in soil samples at each location is not surprising because F. culmorum is a soil-inhabiting fungus (Wagacha and Muthomi, 2007). Besides FHB this pathogen is also causing seedling blight and foot rot. Inter-simple sequence repeat (ISSR) analysis carried out by Mishra et al. (2003) showed a high degree of intra-specific polymorphisms within F. culmorum isolates from different regions and hosts. So probably the F. culmorum isolates on wheat ears in July were not from the same origin of those found in soil samples in November.

On weed samples and crop residues the diversity in species was much larger. On weed samples, mainly $M$. nivale was retrieved, whereas $F$. avenaceum and $F$. graminearum were nearly to the same extent detected on weed samples. The species $M$. nivale and $F$. graminearum were also important species in Flanders in 2007 and 2008 (Audenaert et al., 2009). In 2009 the presence of $F$. avenaceum fluctuated between $0 \%$ in Koksijde and $19.35 \%$ in Zwevegem, whereas F. avenaceum was absent at all locations in 2008 (Audenaert et al., 2009). The importance of $F$. graminearum is in accordance with the reports from other European surveys that point to an increase in the importance of $F$. graminearum as a major pathogen of wheat in temperate climates. The causal factors for this shift have not been elucidated, but an increase in maize production has been suggested to play an important role. Climatic changes might favour the propagation of $F$. graminearum over F. culmorum, as the former species has a higher temperature optimum (Waalwijk et al., 2003). On crop residues, F. graminearum and F. culmorum were the most important species detected. This is in line with the expectations since only risk plants, mainly maize residues, were sampled. Maize residues are important host plants for F. graminearum, which has the capacity to survive on maize stubble. Champeil et al. (2004) concluded that the highest levels of $F$. graminearum contamination are recorded on grains harvested from wheat crops following maize in rotation.

Within the growing season some remarkable differences between the primary inoculum on weeds, crop residues and soil samples and the FHB population in wheat ears were observed. On weed samples and crop residues $F$. poae was a rather marginal species (except in Bottelare and Zwalm), but on wheat ears, in July 2009, the presence of this species varied between $0 \%$ and $39 \%$. So, gramineous weeds and crop residues are probably not the source 
of the F. poae inoculum. Whereas in 2007 and 2008 F. poae was a dominant species, in the present study this species represented only a small part of the final FHB population found on wheat ears. The fact that F. poae is more prominent in 2007 and 2008, years with respectively high and moderate infection pressure, would be a consequence of its nature as a secondary pathogen, colonizing the weakened ears already infected by other more aggressive FHB pathogens (Audenaert et al., 2009). F. avenaceum, completely absent in 2008 (Audenaert et al., 2009), was quite important on weed samples and crop residues, but was not meaningful on wheat ears. The number of $M$. nivale species also decreased during the season.

On gramineous weeds, crop residues and as well on wheat, most pathogens appeared as single species, but there were much more interactions in July on wheat than in November in the primary inoculum. This is in line with the observations of Xu et al. (2005), who observed two-pathogen interactions particularly in harvest samples. F. poae was frequently detected in association, especially with $F$. graminearum. This is in accordance with the hypothesis of Audenaert et al. (2009) that $F$. poae merely acts as a secondary invader. So the population of the primary Fusarium inoculum seems te be less complex than the FHB population on wheat.

In conclusion, this study has demonstrated that the FHB population was characterized by a large variability and complexity and a differential population composition at each location and type of sample (weeds, crop residues, soil and wheat ears). The FHB population in July was more complex than the primary inoculum in December, both the number of different species and the number of interactions rose during the growing season. At two of the eight locations, a good relationship between the primary Fusarium inoculum and the FHB population on wheat ears at the end of the growing season was observed. In Poperinge the population on weeds was similar to the population on wheat ears and in Zwevegem the population on weeds and crop residues was similar to the population on wheat ears. At the other locations some important differences were observed. It is possible that ascospores picked up by turbulent wind currents from other fields contributed to the FHB population or that condia present in weed samples, crop residues and soil samples could not reach the wheat ears. Our findings highlight the importance of frequent surveys of field epidemics to identify FHB population. In this study we focused on the species composition of the primary Fusarium inoculum in November and the FHB population on wheat ears in July and therefore studied the five most important Fusarium species. There seems to be no clear relationship between the species composition of the primary inoculum on weeds, crop residues and soil samples and the FHB population on wheat ears. A totally different research goal is the study of the genetic diversity between Fusarium isolates from different regions or hosts (weeds, crop residues and wheat). The analysis of the genetic structure, 
reproductive behavior and biogeographic structure of the Fusarium species should be done with molecular markers such as random amplified polymorphic DNA (RAPD), simplesequence repeat (SSR) and variable number of tandem repeats (VNTR), which have been employed over the past years and would contribute to our understanding of genetic variability between Fusarium isolates Zhang et al. (2010); Dinolfo et al. (2010). Additional studies are required to see to what extent the population in November contributes to the final population on wheat ears if the observed changes in the Fusarium population during the season prove to be consistent; therefore the same experimental design will be repeated in the coming years.

\section{Acknowledgements}

Kris Audenaert, a postdoctoral fellow of the Research Fund of Ghent University College, coordinated this study. Willem Waegeman is supported as a postdoc by the Research FoundationFlanders (FWO Flanders). This work was financially supported by the Agency for Innovation by Science and Technology, project 70575 (IWT,Brussels, Belgium). We thank the LCG Trial Network for putting the wheat samples at our disposal.

\section{Bibliography}

Audenaert, K., Van Broeck, R., De Witte, F., Heremans, B., Messens, K., Höfte, M., Haesaert, G., 2009. Fusarium head blight(FHB) in Flanders: population diversity, interspecies associations and DON contamination in commercial winter wheat varieties. Eur J Plant Pathol 125, 445-458.

Brennan, J.M., Fagan, B., van Maanen, A., Cooke, B.M., Doohan, F.M., 2003. Studies on in vitro growth and pathogenicity of european Fusarium fungi. Eur J Plant Pathol 109, $577-587$.

Castellá, G., Bragulat, M.R., Rubiales, M.V., Cabañes, F.J., 1997. Malachite green agar, a new selective medium for Fusarium spp. Mycopathologia 137, 173-178.

Champeil, A., Doré, T., Fourbet, J.F., 2004. Fusarium head blight: epidemiological origin of the effects of cultural practices on head blight attacks and the production of mycotoxins by Fusarium in wheat grains. Plant Sci 166, 1389-1415.

Demeke, T., Clear, R.M., Patrick, S.K., Gaba, D., 2005. Species-specific PCR-based assays for the detection of Fusarium species and a comparison with the whole seed agar plate method and trichothecene analysis. Int J Food Microbiol 103, 271-284. 
Dinolfo, M., Stenglein, S.A., Moreno, M., Nicholson, P., Jennings, P., Salerno, G., 2010. ISSR markers detect high genetic variation among Fusarium poae isolates from Argentina and England. Eur J Plant Pathol 127, 483-491.

Hudec, K., Muchová, D., 2010. Influence of temperature and species origin on Fusarium spp. and Microdochium nivale pathogenicity to wheat seedlings. Plant Protect Sci 46, $59-65$.

Isebaert, S., De Saeger, S., Devreese, R., Verhoeven, R., Maene, P., Heremans, B., Haesaert, G., 2009. Mycotoxin-producing Fusarium Species Occurring in Winter Wheat in Belgium (Flanders) During 2002-2005. J Phytopathol 157, 108-116.

Köhl, J., de Haas, B.H., Kastelein, P., Burgers, S.L.G.E., Waalwijk, C., 2007. Population Dynamics of Fusarium spp. and Microdochium nivale in crops and crop residues of winter wheat. Phytopathology 97, 971-978.

Koch, H.J., Pringas, C., Maerlaender, B., 2006. Evaluation of environmental and management effects on Fusarium head blight infection and deoxynivalenol concentration in the grain of winter wheat. Eur J Agron 24, 357-366.

Kosiak, B., Torp, M., Skjerve, E., Thrane, U., 2003. The prevalence and distribution of Fusarium species in Norwegian cereals: a survey. Acta Agriculturae Scandinavica Section B-Soil and Plant Science 53, 168-176.

Leonard, K.J., Bushnell, W.R., 2003. Fusarium Head Blight of Wheat and Barley. APS Press, St. Paul, Minnesota. 512p.

Lukanowski, A., L., L., Sadowski, C., 2008. First report on the occurrence of Fusarium langsethiae isolated from wheat kernels in Poland. Plant Dis 92, 488-488.

Mishra, P., Fox, R., Culham, A., 2003. Inter-simple sequence repeat and aggressiveness analyses revealed high genetic diversity, recombination and long-range dispersal in $\mathrm{Fu}$ sarium culmorum. Ann appl Biol 143, 291-301.

Pereyra, S.A., Dill-Macky, R., 2008. Colonization of the residues of diverse plant species by Gibberella zeae and their contribution to fusarium head blight inoculum. Plant Dis $92,800-807$.

Saghai-Maroof, M.A., Soliman, K.M., Jorgensen, R.A., Allard, R.W., 1984. Ribosomal DNA spacer-length polymorphisms in barley: Mendelian inheritance, chromosomal location, and population dynamics. Proc Natl Acad Sci U S A 81, 8014-8018.

Schaafsma, A.W., Hooker, D.C., 2007. Climatic models to predict occurrence of Fusarium toxins in wheat and maize. Int J Food Microbiol 119, 116-125. 
Torp, M., Nirenberg, H., 2004. Fusarium langsethiae sp. nov. on cereals in Europe. Int J Food Microbiol 95, 247-256.

Waalwijk, C., Kastelein, P., de Vries, I., Kerényi, Z., van der Lee, T., Hesselink, T., Köhl, J., Kema, G., 2003. Major changes in Fusarium spp. in wheat in the Netherlands. Eur J Plant Pathol 109, 743-754.

Wagacha, J.M., Muthomi, J.W., 2007. Fusarium culmorum: Infection process, mechanisms of mycotoxin production and their role in pathogenesis in wheat. Crop Prot 26, 877-885.

Weeda, E., Adema, F.A.C.B., Van Der Meijden, R., 1983. Flora van Nederland. WoltersNoordhoff, Groningen. 20 edition. 583p.

Xu, X.M., Parry, D.W., Nicholson, P., Thomsett, M.A., Simpson, Edwards, S.G., Cooke, B.M., Doohan, F.M., Brennan, J.M., Moretti, A., Tocco, G., Mule, G., Hornok, L., Giczey, G., Tatnell, J., 2005. Predominance and association of pathogenic fungi causing Fusarium ear blight in wheat in four European countries. Eur J Plant Pathol 112, $143-154$.

Zhang, Z., Zhang, H., van der Lee, T., Chen, W., Arens, P., Xu, J., Xu, J.S., Yang, L.J., Yu, D.Z., Waalwijk, C., Feng, J., 2010. Geographic substructure of Fusarium asiaticum isolates collected from barley in China. Eur J Plant Pathol 127, 239-248. 